\title{
Plasticity is a topological business
}

\section{The Topological Origin of the Peierls-Nabarro Barrier}

Authors: Brook J. Hocking, Helen S. Ansell, Randall D. Kamien, and Thomas

Machon

arXiv: 2103.02055 (2021)

\section{Recommended with a Commentary by Brian Skinner, Ohio State University}

Generally speaking, it's much easier to make a plastic deformation in a crystal than one might expect. Plasticity is the degree to which a material deforms permanently under stress, and in a crystal this involves causing one lattice plane to slip with respect to another. Thus, a naive estimate for the critical force required to make a plastic deformation would involve calculating the cumulative force of the atomic interactions between the two neighboring planes. But in fact the necessary force is much smaller because of the presence of dislocations: defects in the crystal at which a perpendicular plane of atoms comes to an end. These dislocations can glide along the slip direction, providing easy paths to plasticity (see Fig. 1).

A common analogy for dislocation-mediated plasticity is to imagine the process of moving a heavy rug across a floor. This process requires much less applied force if one simply makes a wrinkle in the rug and pushes it from one end of the rug to another. It is the analogous dislocation glide process that enables, say, the puny human arm of a blacksmith to mold the shape of a hot piece of iron: the heating and hammering induces dislocations in the iron that can be easily pushed around.

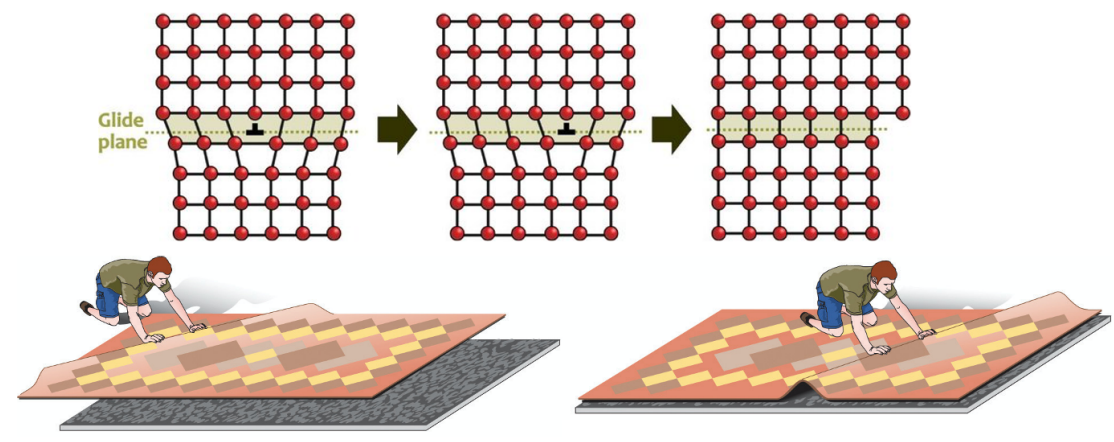

Figure 1: (Above) Plastic deformation of a crystal through dislocation glide (from [1]). (Below) Moving a heavy carpet by pushing a wrinkle (from [2]).

A key materials science question, then, is how much shear stress must be applied in order to cause dislocation glide. Equivalently, one can ask about the corresponding energy barrier. 
A tantalizing question is whether this energy barrier is necessarily finite, or whether one could imagine a crystal for which dislocations glide freely with no energy barrier at all.

The first attempt to address this question came from Peierls, who in 1940 described the elastic stresses surrounding a dislocation using continuum elasticity theory [3]. Of course, in the continuum description there can be no energy barrier for dislocation glide, since without a discrete atomic lattice the elastic energy does not depend on the dislocation position. Peierls could therefore make only a general estimate of the associated energy barrier, but the formula he presented is now a canonical result in engineering textbooks, known as the "Peierls stress." (Or, following later refinements by Nabarro [4], as the "Peierls-Nabarro barrier.") The exact description of the Peierls-Nabarro barrier has received occasional updates in the 80 years since $[5]$.

In the recommended paper [6], the authors reconsider the origin of the Peierls-Nabarro barrier from a topological perspective. They show that, at least in 2D crystals, the PeierlsNabarro can be interpreted as an energy gap between topologically distinct sectors, and therefore that it must be finite as an inevitable consequence of the crystalline order.

In some sense adopting a topological perspective on this problem is natural, since a dislocation itself is a topological object (with a "topological charge" defined by the corresponding Burgers vector). But the authors' result proceeds not so much from the topology of the dislocation as from the topology of the ground state manifold of the crystal itself.

The paper starts by considering the simplified case of a smectic state, for which the ground state has a layered modulation of density $\rho=\rho_{0}+\delta \rho \cos \Phi$. The amplitude $\delta \rho$ is the order parameter of the smectic phase, while the phase field $\Phi=\mathbf{k} \cdot \mathbf{x}+\phi$ (where $\mathbf{k}$ is the wave vector of the density modulation) describes the oscillation of density in space. In the ground state of the smectic state, the phase field is characterized by only two variables: the phase $\phi$ of the density oscillation and the orientation angle $\theta$ of the constant-density planes (see Fig. 2).

The physical system is $2 \pi$-periodic in the phase $\phi$, but only $\pi$-periodic in the orientation $\theta$, with the twist that $(\theta=0, \phi)$ is equivalent to $(\theta=\pi,-\phi)$. Consequently, the space of angles $(\theta, \phi)$ that may be adopted by the ground state (the ground state manifold) has the topology of a Klein bottle.

The ground state manifold admits two basic types of topological defects: dislocations and disclinations. Dislocations are points in the phase field around which any closed path involves a winding of the phase by $2 \pi$. Disclinations are points at which the orientation angle abruptly flips, so that a closed path around a disclination involves a jump from $(\theta, \phi)$ to $(\theta \pm \pi,-\phi)$. In order to establish the topological inevitability of the Peierls-Nabarro barrier, the authors show two things:

1. Disclinations can reside only at specific values of the phase $(\phi=0$ or $\phi=\pi)$. Otherwise either the density $\rho$ becomes discontinuous, or the order parameter $\delta \rho$ must vanish.

2. A dislocation can always be decomposed into two opposite disclinations. This can be shown by smoothly deforming the path around a dislocation, as shown in Fig. 2.

Together, these two conditions imply that dislocations cannot move continuously, since they are made of disclinations and disclinations are pinned in place (by an energy barrier). 

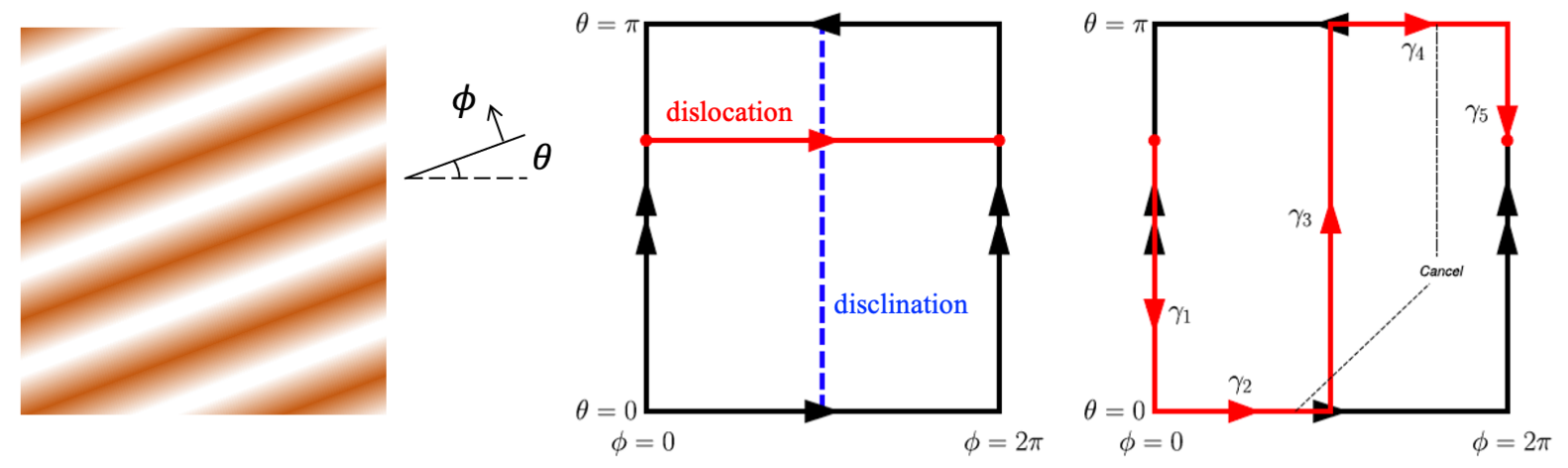

Figure 2: (Left) The modulation of density in the smectic ground state (dark and light colored regions), showing the orientation angle $\theta$ and the phase $\phi$. (Middle) The ground state manifold in the space of $\phi$ and $\theta$, with the boundary conditions indicated by black arrows. Tracing a path around a dislocation involves a $2 \pi$ winding of the phase $\phi$. A path around a disclination involves a $\pm \pi$ change in orientation angle $\theta$. (Right) The path around a dislocation can be continuously deformed until it consists of two opposite disclinations. Adapted from [6].

Only a vanishing of the order parameter $\delta \rho$, which would correspond to melting of the crystal, would enable dislocations to glide freely.

After establishing the topological origin of the Peierls-Nabarro barrier for the smectic case, the authors analyze the case of $2 \mathrm{D}$ lattices, which have not one phase $\phi$ but two. They describe the corresponding ground state manifolds for each of the five Bravais lattices in two dimensions, and catalog the allowed locations (in the phase field) of disclinations. The upshot of this analysis is the same: that dislocations are inevitably composed of disclinations, which can reside only at specific locations in the phase field, and so a Peierls-Nabarro barrier is inevitable. But by defining defects in terms of the phase field, the authors' description provides a convenient and simple categorization of disclinations, which for some lattice types can be hard to draw or intuit in terms of atomic positions alone.

In one sense, there are no obvious new phenomena implied by the paper, since its purpose is only to recast known concepts (e.g., the Peierls-Nabarro barrier) in a new light. But it is invigorating to see this classic result rephrased in topological language, and derived using only the topology of the ground state manifold. The authors' line of thinking may spark more insights related to topological defects in two-dimensional systems, or even in three dimensions (where defects are line-like and can tangle with each other).

\section{References}

[1] P. Cordier and A. Goryaeva, Multiscale modeling of the mantle rheology, European Research Council (2018).

[2] B. Averill and P. Eldredge, General chemistry: Principles, patterns, and applications, DC: Saylor Academy (2015). 
[3] R. Peierls, "The size of a dislocation," Proceedings of the Physical Society 52, 34 (1940).

[4] F. R. N. Nabarro, "Dislocations in a simple cubic lattice," Proceedings of the Physical Society 59, 256 (1947).

[5] F. R. N. Nabarro, "Fifty-year study of the Peierls-Nabarro stress," Materials Science and Engineering: A 234, 67 (1997).

[6] B. J. Hocking, H. S. Ansell, R. D. Kamien, and T. Machon, "The Topological Origin of the Peierls-Nabarro Barrier," arXiv:2103.02055 (2021). 